\title{
Exponential Synchronization for Second-Order Nodes in Complex Dynamical Network with Communication Time Delays and Switching Topologies
}

\author{
Miao Yu, ${ }^{1,2}$ Weipeng Shang, ${ }^{1,2}$ and Zhigang Chen ${ }^{1,2}$ \\ ${ }^{1}$ School of Mechanical-Electronic and Automobile Engineering, Beijing University of Civil Engineering and Architecture, \\ Beijing 100044, China \\ ${ }^{2}$ Beijing Key Laboratory for Service Performance Guarantee of Urban Rail Transit Vehicles, Beijing 100044, China \\ Correspondence should be addressed to Miao Yu; olivermiaoer@163.com
}

Received 8 July 2016; Revised 2 January 2017; Accepted 9 January 2017; Published 13 February 2017

Academic Editor: Petko Petkov

Copyright (C) 2017 Miao Yu et al. This is an open access article distributed under the Creative Commons Attribution License, which permits unrestricted use, distribution, and reproduction in any medium, provided the original work is properly cited.

\begin{abstract}
This paper is devoted to the study of exponential synchronization problem for second-order nodes in dynamical network with time-varying communication delays and switching communication topologies. Firstly, a decomposition approach is employed to incorporate the nodes' inertial effects into the distributed control design. Secondly, the sufficient conditions are provided to guarantee the exponential synchronization of second-order nodes in the case that the information transmission is delayed and the communication topology is balanced and arbitrarily switched. Finally, to demonstrate the effectiveness of the proposed theoretical results, it is applied to the typical second-order nodes in dynamical network, as a case study. Simulation results indicate that the proposed method has a high performance in synchronization of such network.
\end{abstract}

\section{Introduction}

A complex dynamical network consists of a number of nodes and links between them. Complex networks exist in many fields of science, engineering, and society and have attracted much attention in recent years $[1,2]$. As one of the most important collective behaviors, synchronization phenomena have been a topic of research. This topic occurs in physical science field and mathematics field for quite some time [35]. Several books and reviews $[6,7]$ which deal with this topic have also appeared. Such applications are pervasive and include clock synchronization in complex networks [8-10], coordination of unmanned air vehicles [11], and allocation of network resources fairly [12].

During the past decades, there are lots of results about synchronization of dynamical networks using graph theory and matrix theory. In [11] a Vicsek model for synchronization of dynamical network has been proposed. Also synchronization between multiagent systems with first-order integral plants when the communication topologies are balanced graphs has been studied in [5]. Extensions to the works of $[5,11]$ have been presented in [13] where the control conditions for synchronization have been broadened. Using the Lyapunov control theory, the synchronization problem of multiagent systems with time-varying communication topologies has been investigated in [14]. A number of research results about synchronization in complex networks have been put forward in $[15,16]$. When the nodes in the dynamical network are high order integral plants, the synchronization problem becomes more challenging. A general way to synchronize the dynamical network has been presented and applied to multiple second-order integral plants in complex dynamical network in [17-19]. Recognition issue for unknown system parameters and topology of uncertain general complex dynamical networks with nonlinear couplings and time-varying delay is investigated through generalized outer synchronization [20]. A pinning controller is designed for cluster synchronization of complex dynamical networks with semi-Markovian jump topology [21]. Improved delaydependent stability criteria for continuous systems with two 
additive time-varying delay components are analyzed [22]. It is well known that inertia is an important parameter in the controller design. In all the work mentioned above, each inertial node is considered to be the same. So far, there are few research works about synchronization of different inertial nodes in dynamical network. However, in real world, when there are communication time delays between inertial nodes, the synchronization problem will become more and more complicated. Synchronization of dynamical network with fixed topologies and undirected time delays has been studied in [23]. Further study of synchronization of dynamical network with switching topologies has been investigated in [24]. The time delay problem of directed communication network has been also considered in [14, 17, 24, 25].

In real complex dynamical network, time-varying communication topologies, communication time delays, and inertial nodes are three important factors to achieve synchronization. The existing literature fails to consider time-varying communication topologies, communication time delays, and inertial nodes at the same time. This paper takes these three factors into account simultaneously and explores the problem of synchronization between different second-order inertial nodes. The objective of this paper is to analyze the synchronization properties for a complex dynamical network with second-order nodes, time-varying communication delays, and switching communication topologies. Based on this model, several sufficient conditions for exponential synchronization stability are obtained by employing the Lyapunov functional method.

The remainder of this paper is organized as follows. A generalized model of complex dynamical network with second-order nodes, time-varying communication delays, and switching communication topologies is introduced and some useful mathematical preliminaries are given in Section 2. The method of decomposition of dynamical network is studied in Section 3. Section 4 deals with several criteria for synchronization stability. In this section, some sufficient conditions are obtained to achieve exponential synchronization for second-order nodes in dynamical network. To illustrate the theories obtained in Section 4, numerical examples with specific communication delays and switching topologies are used in Section 5. Finally, in Section 6, we summarize our results.

\section{Model Formulation and Mathematical Preliminaries}

2.1. Model Description. Consider a dynamical network consisting of diffusively coupled identical nodes, with each node being a second-order dynamical unit. The state equations of each node are described by

$$
M_{i} \ddot{x}_{i}=u_{i}
$$

that is,

$$
\begin{aligned}
\dot{x}_{i} & =v_{i}, \\
M_{i} \dot{v}_{i} & =u_{i},
\end{aligned}
$$

where $x_{i} \in \mathbb{R}^{m}$ are the position vectors; $v_{i} \in \mathbb{R}^{m}$ stand for the velocity vectors; $M_{i} \in \mathbb{R}^{m \times m}$ are symmetric positive definite matrices; and $u_{i} \in \mathbb{R}^{m}$ are the control inputs. Let $M=$ $\operatorname{diag}\left\{M_{1}, M_{2}, \ldots, M_{n}\right\} \in \mathbb{R}^{n m \times n m}, x=\left[x_{1}^{\mathrm{T}}, x_{2}^{\mathrm{T}}, \ldots, x_{n}^{\mathrm{T}}\right]^{\mathrm{T}} \in$ $\mathbb{R}^{n m}, v=\left[v_{1}^{\mathrm{T}}, v_{2}^{\mathrm{T}}, \ldots, v_{n}^{\mathrm{T}}\right]^{\mathrm{T}} \in \mathbb{R}^{n m}, u=\left[u_{1}^{\mathrm{T}}, u_{2}^{\mathrm{T}}, \ldots, u_{n}^{\mathrm{T}}\right]^{\mathrm{T}} \in$ $\mathbb{R}^{n m}$.

According to (2), for $n$ th, there is

$$
\begin{aligned}
\dot{x} & =v, \\
M \dot{v} & =u .
\end{aligned}
$$

Suppose that there exists a communication time delay $\tau(t)$ between nodes $i$ and $j$; the time-varying delay $\tau(t)$ satisfies any one assumption of the following:

(A1) $0 \leq \tau(t) \leq h, \dot{\tau}(t) \leq r<1$;

(A2) $0 \leq \tau(t) \leq h$,

where $h>0$. For each node $i$ in the dynamical network, the control law based on neighbor's messages is used. The control law can be expressed as follows:

$$
\begin{aligned}
& u_{i}(t)=\sum_{j \in \aleph_{i}(\sigma(t))}\left[b a_{i j} \Lambda\left(v_{j}(t-\tau(t))-v_{i}(t-\tau(t))\right)\right. \\
& \left.\quad+k a_{i j} \Lambda\left(x_{j}(t-\tau(t))-x_{i}(t-\tau(t))\right)\right]
\end{aligned}
$$

where $b>0, k>0 ; \Lambda \in \mathbb{R}^{m \times m}$ is a positive definite diagonal matrix; $\sigma:[0,+\infty) \rightarrow \wp=\{1, \ldots, N\}$ ( $N$ denotes the total number of all possible directed graphs) is a switching signal of communication topology; and $\aleph_{i}(\sigma(t))$ denotes the neighbor net of node $i$ in graph $G_{\sigma(t)}$. In the following, in order to discuss conveniently, we abbreviate $\sigma(t)$ as $\sigma$.

According to (3) and (4), the dynamical network model can be acquired as follows:

$$
\begin{aligned}
\dot{x}= & v, \\
M \dot{v}= & -b\left(L^{\sigma} \otimes \Lambda\right) v(t-\tau(t)) \\
& -k\left(L^{\sigma} \otimes \Lambda\right) x(t-\tau(t)),
\end{aligned}
$$

where $L^{\sigma} \in \mathbb{R}^{n \times n}$ is Laplacian matrix of graph $G_{\sigma}$. Suppose that $L^{\sigma}$ has the following properties:

(A3) $\operatorname{rank}\left(L^{\sigma}\right)=n-1$;

(A4) $L^{\sigma} \mathbf{1}_{n}=0$.

Remark 1. If (A3) holds, then graph $G_{\sigma}$ is a strongly connected graph. And if graph is equilibrium diagram, then (A4) holds [5]. Let $\mathbb{C}\left([-\tau(t), 0], \mathbb{R}^{n m}\right)$ be a continuous vectorvalued function in Banach space. For any given

$$
\Phi=\left[\Phi_{1}^{\mathrm{T}}, \Phi_{2}^{\mathrm{T}}\right]^{\mathrm{T}} \in \mathbb{C}^{2}
$$

$$
\text { where } \Phi_{i} \in \mathbb{C}(i=1,2), t_{0} \in \mathbb{R} \text {, }
$$

when $t>t_{0}-\tau(t)$, there exists an only solution $x\left(t ; t_{0}, \Phi_{1}\right)$, $v\left(t ; t_{0}, \Phi_{2}\right)$ of (5). We define two manifolds: $\Gamma_{1}=\left\{\left[x_{1}^{\mathrm{T}}\right.\right.$, $\left.\left.x_{2}^{\mathrm{T}}, \ldots, x_{n}^{\mathrm{T}}\right]^{\mathrm{T}} \in \mathbb{R}^{n m} ; x_{i}=x_{j}, i, j \in H\right\}, \Gamma_{2}=\left\{\left[v_{1}^{\mathrm{T}}\right.\right.$, $\left.\left.v_{2}^{\mathrm{T}}, \ldots, v_{n}^{\mathrm{T}}\right]^{\mathrm{T}} \in \mathbb{R}^{n m} ; v_{i}=v_{j}, i, j \in H\right\}$. 
Definition 2. For $\Phi_{i} \in \mathbb{C}(i=1,2)$ and $t_{0} \in \mathbb{R}$, if there are some constants $\varepsilon_{1}>0, \varepsilon_{2}>0, \eta_{1}>0$, and $\eta_{2}>0$, such that

$$
\begin{gathered}
\left\|x_{i}-x_{j}\right\| \leq \eta_{1} e^{-\varepsilon_{1}\left(t-t_{0}\right)}, \quad i, j \in H, \\
\left\|v_{i}-v_{j}\right\| \leq \eta_{2} e^{-\varepsilon_{2}\left(t-t_{0}\right)}, \quad i, j \in H
\end{gathered}
$$

for all $t \geq t_{0}$ and $\tau(t) \in[0, h]$ hold; then manifold $\Gamma_{1}$ and manifold $\Gamma_{2}$ are exponential stable.

Remark 3. To discuss in a simple way, we consider how to achieve the synchronization problem of $x_{i}-x_{j} \rightarrow 0, \dot{x}_{i}-\dot{x}_{j} \rightarrow$ $0,(i, j \in H)$. By the rational selection of state information, the results in this paper can be applied to many practical problems, such as synchronization problem and formation control. By altering the control law (4), we can achieve $\dot{x}_{i}-$ $\dot{x}_{j} \rightarrow 0, x_{i}-x_{j} \rightarrow \delta_{i j},(i, j \in H)$, where $\delta_{i j} \in \mathbb{R}^{m}$ denotes the mutual distance between node $i$ and node $j$. Let $\delta_{i} \in \mathbb{R}^{m}$ be a constant and the control input is

$$
\begin{aligned}
& u_{i}(t)=\sum_{j \in \aleph_{i}(\sigma)}\left[b a_{i j} \Lambda\left(v_{j}(t-\tau(t))-v_{i}(t-\tau(t))\right)\right. \\
& \quad+k a_{i j} \Lambda\left(\left(x_{j}(t-\tau(t))-\delta_{j}\right)\right. \\
& \left.\left.\quad-\left(x_{i}(t-\tau(t))-\delta_{i}\right)\right)\right] ;
\end{aligned}
$$

then we can get $x_{i}(t-\tau(t))-\delta_{i} \rightarrow x_{j}(t-\tau(t))-\delta_{j},\left(\delta_{i j}=\delta_{i}-\delta_{j}\right)$, $v_{i} \rightarrow v_{j}$.

2.2. Preliminaries. The interaction topology of a dynamical network of nodes with second-order nodes is represented using a directed graph $G=(V, E, A)$ with the set of nodes $V=\left\{v_{1}, v_{2}, \ldots, v_{n}\right\}$, the set of directed edges is $E \subseteq V \times$ $V$, and the adjacency matrix is $A=\left[a_{i j}\right] \in \mathbb{R}^{n \times n} . H \in$ $\{1,2, \ldots, n\}$ is the set of node subscripts; $e_{i j}=(i, j)$ denotes the directed edge from node $i$ to node $j$. The elements in adjacency matrix $A=\left[a_{i j}\right] \in \mathbb{R}^{n \times n}$ satisfy $a_{i i}=0, a_{i j}>0$ (if and only if $e_{i j} \in E$ ). The set of neighbors of node $i$ is $\aleph_{i}=\{j \in H:(i, j) \in E\}$. If there is $d_{i}=\sum_{j \in \aleph_{i}} a_{i j}$, $i \in H$, then the degree matrix of a directed graph $G$ can be expressed as $D=\operatorname{diag}\left\{d_{1}, d_{2}, \ldots, d_{n}\right\}$. The Laplacian matrix $L$ is defined by $L \triangleq D-A$. It can be shown that, using the Gershgorin disc theorem [12], all of the eigenvalues of $L$ have a nonnegative real part. Furthermore, if $G$ is undirected, then the Laplacian matrix of $G$ is symmetric and there is $L=B B^{\mathrm{T}}$, which means that $L$ is positive semidefinite. The in-degree and out-degree of node $V_{i}$ can be defined as $d_{\text {in }}\left(v_{i}\right)=\sum_{j=1}^{n} a_{j i}$ and $d_{\text {out }}\left(\nu_{i}\right)=\sum_{j=1}^{n} a_{i j}$, respectively. If $d_{\text {in }}\left(\nu_{i}\right)=\sum_{j=1}^{n} a_{j i}=$ $d_{\text {out }}\left(v_{i}\right)=\sum_{j=1}^{n} a_{i j}$, then node $v_{i}$ is an equilibrium point. If there exists a direct graph between nodes in graph $G$, then $G$ is a strongly connected graph. In addition, $I_{n}$ denotes $n$-order identity matrix. $\mathbf{1}_{n}$ denotes $n$-dimensional column vector of which all the elements are $1 . \lambda_{\max }(S)$ and $\lambda_{\text {min }}(S)$ denote the maximum and minimum eigenvalue of real symmetric matrix $S$, respectively. $\|\cdot\|$ denotes the Euclidean norm and $\otimes$ denotes Kronecker product. The Dini time derivative of continuous function $V: \mathbb{R} \rightarrow \mathbb{R}$ can be defined as $D^{+} V=$ $\lim _{h \rightarrow 0^{+}} \sup ((V(t+h)-V(t)) / h)$.

\section{Decomposition of Dynamical Network}

We can decompose the dynamical network (5) into cluster subsystem and formation subsystem according to the method mentioned in [15]. Take the decomposition transformation:

$$
z=S x
$$

$S \in \mathbb{R}^{n m \times n m}$ is the transformation matrix, which is defined by

$$
S=\left[\begin{array}{ccccc}
\varphi_{1} & \varphi_{2} & \varphi_{3} & \cdots & \varphi_{n} \\
I_{m} & -I_{m} & 0 & \cdots & 0 \\
0 & I_{m} & -I_{m} & \ddots & \vdots \\
\vdots & \vdots & \ddots & \ddots & 0 \\
0 & \cdots & 0 & I_{m} & -I_{m}
\end{array}\right]
$$

where

$$
\begin{aligned}
\varphi_{i} & =\left(\sum_{j=1}^{n} M_{j}\right)^{-1} M_{i} \in \mathbb{R}^{m \times m}, \\
z & =\left[z_{1}^{\mathrm{T}}, z_{e}^{\mathrm{T}}\right]^{\mathrm{T}} \in \mathbb{R}^{n m}
\end{aligned}
$$

is a new variable, in which

$$
\begin{aligned}
& z_{1}=\left(\sum_{j=1}^{n} M_{j}\right)^{-1} \sum_{i=1}^{n} M_{i} x_{i} \in \mathbb{R}^{m}, \\
& z_{e}=\left[x_{1}^{\mathrm{T}}-x_{2}^{\mathrm{T}}, x_{2}^{\mathrm{T}}-x_{3}^{\mathrm{T}}, \ldots, x_{n-1}^{\mathrm{T}}-x_{n}^{\mathrm{T}}\right]^{\mathrm{T}} \in \mathbb{R}^{m(n-1)} .
\end{aligned}
$$

According to (9), the dynamical network model (5) can be written as

$$
\begin{aligned}
S^{-\mathrm{T}} M S^{-1} \ddot{z}= & -b S^{-\mathrm{T}}\left(L^{\sigma} \otimes \Lambda\right) S^{-1} \dot{z}(t-\tau(t)) \\
& -k S^{-\mathrm{T}}\left(L^{\sigma} \otimes \Lambda\right) S^{-1} z(t-\tau(t)),
\end{aligned}
$$

where $S^{-1} \in \mathbb{R}^{n m \times n m}$ has the following form:

$$
S^{-1}=\left[\begin{array}{ccccc}
I_{m} & \phi_{2} & \phi_{3} & \ldots & \phi_{n} \\
I_{m} & \phi_{2}-I_{m} & \phi_{3} & \ldots & \phi_{n} \\
I_{m} & \phi_{2}-I_{m} & \phi_{3}-I_{m} & \ddots & \vdots \\
\vdots & \vdots & \ddots & \ddots & \phi_{n} \\
I_{m} & \phi_{2}-I_{m} & \ldots & \phi_{n-1}-I_{m} & \phi_{n}-I_{m}
\end{array}\right]
$$

where $\phi_{i}=\sum_{j=i}^{n} \varphi_{j}$. From (14), we have

$$
S^{-\mathrm{T}} M S^{-1}=\left[\begin{array}{cc}
M_{c} & 0 \\
0 & M_{e}
\end{array}\right],
$$

where $M_{c}=\sum_{j=1}^{n} M_{j} \in \mathbb{R}^{m \times m}$ and $M_{e} \in \mathbb{R}^{(n-1) m \times(n-1) m}$. 
Because the row sum of matrix $L^{\sigma}$ is zero, then we have

$$
S^{-\mathrm{T}}\left(L^{\sigma} \otimes \Lambda\right) S^{-1}=\left[\begin{array}{cc}
0 & H^{\mathrm{T}} \\
0 & L_{e}^{\sigma}
\end{array}\right],
$$

where $H \in \mathbb{R}^{(n-1) m \times m}$; the $j$ th element of $H$ is

$$
\begin{aligned}
H_{j}=-\left(\sum_{k=j+1}^{n} \sum_{i=1}^{n} L_{i k}^{\sigma}\right) \Lambda \in \mathbb{R}^{m \times m}, & \\
& j \in\{1,2, \ldots, n-1\} .
\end{aligned}
$$

$(i, j)$ Jordan of $L_{e}^{\sigma} \in \mathbb{R}^{(n-1) m \times(n-1) m}$ is

$$
L_{e, i j}^{\sigma}=\phi_{i+1}^{\mathrm{T}} H_{j}+\sum_{p=i+1}^{n} \sum_{q=j+1}^{n} L_{i k}^{\sigma} \Lambda \in \mathbb{R}^{m \times m} .
$$

It follows from (13), (15), and (16) that the dynamical network model (5) can be decomposed into

$$
\begin{aligned}
& M_{c} \ddot{z}_{1}=-b H^{\mathrm{T}} \dot{z}_{e}(t-\tau(t))-k H^{\mathrm{T}} z_{e}(t-\tau(t)), \\
& M_{e} \ddot{z}_{e}=-b L^{\mathrm{T}} \dot{z}_{e}(t-\tau(t))-k L^{\mathrm{T}} z_{e}(t-\tau(t)) .
\end{aligned}
$$

Theorem 4. Consider the dynamical network (5); its decomposition transformation models are (19) and (20). Suppose that (A3) and (A4) hold; then we have the following.

(1) For any initial condition and given $\{b, k\}$, the centre-ofmass velocity $\dot{z}_{1}(t)$ remains unchanged; that is,

$$
\dot{z}_{1}(t)=\left(\sum_{j=1}^{n} M_{j}\right)^{-1} \sum_{i=1}^{n} M_{i} \dot{x}_{i}\left(t_{0}\right), \quad \forall t \geq t_{0} .
$$

(2) If the delay differential equation

$$
\dot{\varepsilon}(t)=A \varepsilon(t)+B^{\sigma} \varepsilon(t-\tau(t))
$$

is exponential stable for zero solution, then manifold $\Gamma_{1}$ and manifold $\Gamma_{2}$ are exponential stable, where

$$
\begin{aligned}
\varepsilon & =\left[\begin{array}{c}
z_{e} \\
\dot{z}_{e}
\end{array}\right], \\
A & =\left[\begin{array}{cc}
0 & I_{(n-1) m} \\
0 & 0
\end{array}\right], \\
B^{\sigma} & =\left[\begin{array}{cc}
0 & 0 \\
-k M_{e}^{-1} L_{e}^{\sigma} & -b M_{e}^{-1} L_{e}^{\sigma}
\end{array}\right] .
\end{aligned}
$$

Proof. (1) According to (A4), we have $H_{j}=$ $-\left(\sum_{k=j+1}^{n} \sum_{i=1}^{n} L_{i k}^{\sigma}\right) \Lambda=0$. Hence, there is $H=0$. From (19), we can get the conclusion that $M_{c} \ddot{z}_{1}=0$. It is obvious that for any $t \geq t_{0}, \dot{z}_{1}$ is invariable; that is, (21) holds.

(2) According to (20), we have

$$
\ddot{z}_{e}=-b M_{e}^{-1} L_{e}^{\sigma} \dot{z}_{e}(t-\tau(t))-k M_{e}^{-1} L_{e}^{\sigma} z_{e}(t-\tau(t)) .
$$

Furthermore, for the zero solution, if (22) is exponential stable (i.e., there exist constants $\eta_{0}>0$ and $\lambda_{0}>0$ ), then the solution $\varepsilon\left(t_{0}, \Phi\right)(t)$ of $(22)$ on $\left(t_{0}, \Phi\right) \in \mathbb{R} \times \mathbb{C}^{2}$ satisfies $\|\varepsilon\| \leq \eta_{0} e^{-\lambda_{0}\left(t-t_{0}\right)}$. It is clear that manifold $\Gamma_{1}$ and manifold $\Gamma_{2}$ are exponential stable.

\section{Synchronization for Second-Order Nodes in Complex Dynamical Network with Communication Time Delays and Switching Topologies}

Theorem 5. Suppose that (A1) holds. The communication topology $G_{\sigma}$ satisfies (A3) and (A4). Then for given constants $\alpha>0, h>0$, and $r \geq 0$, if there exist positive definite matrices $P, R, U$ in the corresponding dimensions and arbitrary matrices $H_{1}, H_{2}$ satisfying

$$
\left[\begin{array}{cccc}
\Delta_{11} & \Delta_{12} & q_{0} H_{1} & h A^{\mathrm{T}} R \\
\Delta_{12}^{\mathrm{T}} & \Delta_{22} & q_{0} H_{2} & h\left(B^{\sigma}\right)^{\mathrm{T}} R \\
q_{0} H_{1}^{\mathrm{T}} & q_{0} H_{2}^{\mathrm{T}} & -q_{0} R & 0 \\
h R A & h R B^{\sigma} & 0 & -h R
\end{array}\right]<0
$$

where $\Delta_{11}=P A+A^{\mathrm{T}} P+\alpha P+U+H_{1}^{\mathrm{T}}+H_{1}, \Delta_{12}=P B^{\sigma}-H_{1}+H_{2}^{\mathrm{T}}$, $\Delta_{22}=-(1-r) e^{\alpha h} U-H_{2}-H_{2}^{\mathrm{T}}, q_{0}=\left(e^{\alpha h}-1\right) / \alpha$, then system (5), manifold $\Gamma_{1}$, and manifold $\Gamma_{2}$ are exponential stable. The solution of (22) satisfies

$$
\|\varepsilon(t)\| \leq \sqrt{\frac{b_{0}}{a_{0}}} e^{-\alpha\left(t-t_{0}\right) / 2}\left\|\varepsilon_{t_{0}}\right\|_{c},
$$

where

$$
\begin{aligned}
a_{0} & =\lambda_{\text {min }}(P), \\
b_{0} & =\lambda_{\text {max }}(P)+h \lambda_{\text {max }}(U)+\frac{h^{2}}{2} \lambda_{\text {max }}(R), \\
\left\|\varepsilon_{t_{0}}\right\|_{c} & =\sup _{-\tau(t) \leq \theta \leq 0}\left\{\left\|\varepsilon\left(t_{0}+\theta\right)\right\|,\left\|\dot{\varepsilon}\left(t_{0}+\theta\right)\right\|\right\}, \\
\dot{x}_{i}(t) & \longrightarrow \dot{z}_{1}(t)=\left(\sum_{j=1}^{n} M_{j}\right)^{-1} \sum_{i=1}^{n} M_{i} \dot{x}_{i}\left(t_{0}\right),
\end{aligned}
$$

$\forall i \in H$

Proof. Choose the following Lyapunov function:

$$
V(t)=V_{1}(t)+V_{2}(t)+V_{3}(t)
$$

where

$$
\begin{aligned}
& V_{1}(t)=\varepsilon^{\mathrm{T}}(t) P \varepsilon(t), \\
& V_{2}(t)=\int_{-h}^{0} \int_{t+\theta}^{t} \dot{\varepsilon}^{\mathrm{T}}(s) e^{\alpha(s-t)} R \dot{\varepsilon}(s) d s d \theta, \\
& V_{3}(t)=\int_{t-\tau(t)}^{t} \varepsilon^{\mathrm{T}}(s) e^{\alpha(s-t)} U \mathcal{\varepsilon}(s) d s .
\end{aligned}
$$


According to (22), we have

$$
\begin{aligned}
& D^{+} V_{2}(t) \\
& \leq-\alpha \int_{-h}^{0} \int_{t+\theta}^{t} \dot{\varepsilon}^{\mathrm{T}}(s) e^{\alpha(s-t)} R \dot{\varepsilon}(s) d s d \theta \\
& \quad+h \dot{\varepsilon}^{\mathrm{T}}(t) R \dot{\varepsilon}(t)-\int_{t-\tau(t)}^{t} \dot{\varepsilon}^{\mathrm{T}}(s) e^{\alpha(s-t)} R \dot{\varepsilon}(s) d s, \\
& D^{+} V_{3}(t) \\
& =-\alpha \int_{t-\tau(t)}^{t} \varepsilon^{\mathrm{T}}(s) e^{\alpha(s-t)} U \varepsilon(s) d s+\varepsilon^{\mathrm{T}}(t) U \varepsilon(t) \\
& \quad-(1-\dot{\tau}(t)) \varepsilon^{\mathrm{T}}(t-\tau(t)) e^{-\alpha \tau} U \varepsilon(t-\tau(t)) .
\end{aligned}
$$

From Leibniz-Newton formula, we know that

$$
\begin{aligned}
& (1-\dot{\tau}(t)) \varepsilon^{\mathrm{T}}(t-\tau(t)) e^{-\alpha \tau} U \mathcal{\varepsilon}(t-\tau(t)) \\
& \quad \geq(1-r) \varepsilon^{\mathrm{T}}(t-\tau(t)) e^{-\alpha h} U \mathcal{\varepsilon}(t-\tau(t)) .
\end{aligned}
$$

According to (A1) and (32), we have

$$
\begin{aligned}
& (1-\dot{\tau}(t)) \varepsilon^{\mathrm{T}}(t-\tau(t)) e^{-\alpha \tau} U \mathcal{\varepsilon}(t-\tau(t)) \geq(1-r) \\
& \cdot \varepsilon^{\mathrm{T}}(t-\tau(t)) e^{-\alpha h} U \varepsilon(t-\tau(t)), \\
& -\int_{t-\tau(t)}^{t} \dot{\varepsilon}^{\mathrm{T}}(s) e^{\alpha(s-t)} R \dot{\varepsilon}(s) d s-2\left[\varepsilon^{\mathrm{T}}(t) H_{1}\right. \\
& \left.+\varepsilon^{\mathrm{T}}(t-\tau(t)) H_{2}\right] \int_{t-\tau(t)}^{t} \dot{\varepsilon}(s) d s \\
& \leq-\int_{t-\tau(t)}^{t}\left(\xi^{\mathrm{T}}(t) H+\dot{\varepsilon}^{\mathrm{T}}(s) e^{\alpha(s-t)} R\right)\left(e^{\alpha(s-t)} R\right)^{-1} \\
& \cdot\left(H^{\mathrm{T}} \xi(t)+e^{\alpha(s-t)} R \dot{\varepsilon}(s)\right) d s+q_{0} \xi^{\mathrm{T}}(t) \\
& \cdot H R^{-1} H^{\mathrm{T}} \xi(t),
\end{aligned}
$$

where

$$
\begin{aligned}
\xi(t) & =\left[\begin{array}{c}
\varepsilon(t) \\
\varepsilon(t-\tau(t))
\end{array}\right], \\
H & =\left[\begin{array}{l}
H_{1} \\
H_{2}
\end{array}\right] .
\end{aligned}
$$

It follows from (31) and (33) that

$$
\begin{aligned}
& \dot{V}(t)+\alpha V(t) \leq \xi^{\mathrm{T}}(t) \Xi \xi(t) \\
& \quad-\int_{t-\tau(t)}^{t}\left(\xi^{\mathrm{T}}(t) H+\dot{\varepsilon}^{\mathrm{T}}(s) e^{\alpha(s-t)} R\right)\left(e^{\alpha(s-t)} R\right)^{-1} \\
& \quad \cdot\left(H^{\mathrm{T}} \xi(t)+e^{\alpha(s-t)} R \dot{\varepsilon}(s)\right) d s
\end{aligned}
$$

where

$$
\begin{aligned}
\Xi= & {\left[\begin{array}{cc}
\Delta_{11}+h A^{\mathrm{T}} R A & \Delta_{12}+h A^{\mathrm{T}} R B^{\sigma} \\
\Delta_{12}^{\mathrm{T}}+h\left(B^{\sigma}\right)^{\mathrm{T}} R A & \Delta_{22}+h\left(B^{\sigma}\right)^{\mathrm{T}} R B^{\sigma}
\end{array}\right] } \\
& +q_{0} H R^{-1} H^{\mathrm{T}} .
\end{aligned}
$$

According to Schur complement lemma, (25) guarantees $\Xi<$ 0 . It can be obtained from (35) that $D^{+} V(t)+\alpha V(t) \leq 0$. Hence, we have

$$
V(t) \leq \sup _{-\tau(t) \leq s \leq 0} V\left(t_{0}+s\right) e^{-\alpha\left(t-t_{0}\right)} .
$$

From (27) to (29), it can be obtained that

$$
\begin{gathered}
a_{0}\|\varepsilon(t)\|^{2} \leq V(t), \\
\sup _{-\tau(t) \leq s \leq 0} V\left(t_{0}+s\right) \leq b_{0}\left\|\varepsilon_{t_{0}}\right\|_{c}^{2} .
\end{gathered}
$$

Then, according to (37) and (38), we can get (26). It is known from (26) that (22) is exponential stable for zero solution. According to Theorem 4 , when the time delay $\tau(t)$ satisfies (A1), for the dynamical network (5), manifold $\Gamma_{1}$ and manifold $\Gamma_{2}$ are exponential stable. From $x_{i}(t)-x_{j}(t) \rightarrow 0$, we can obtain $z_{1}(t) \rightarrow x_{i}(t), \forall i \in H$. We can also get (28) from (21).

Remark 6. Suppose that (25) is available at $h=h_{0}$. For any $0 \leq r<1$, we can obtain the maximum $h$ by the following steps: Step 1: let $h=h_{0}$; Step 2: to find the matrices $\{P, R, U\}$ satisfy (25). If we find matrices $\{P, R, U\}$, then let $h=h+g_{0}\left(g_{0}\right.$ is the step length). Repeat Step 2; otherwise, quit the whole procedure. $h$ is the permitted maximal time delay.

Theorem 7. Suppose that (A2) holds. The communication topology $G_{\sigma}$ satisfies (A3) and (A4). If $U=0$ and (25) holds, then, for the dynamical network (5), manifold $\Gamma_{1}$ and manifold $\Gamma_{2}$ are exponential stable. The solution of (22) satisfies

$$
\|\varepsilon(t)\| \leq \sqrt{\frac{b_{1}}{a_{0}}} e^{-\alpha\left(t-t_{0}\right) / 2}\left\|\varepsilon_{t_{0}}\right\|_{c},
$$

where

$$
\begin{aligned}
& a_{0}=\lambda_{\text {min }}(P), \\
& b_{1}=\lambda_{\text {max }}(P)+\frac{h^{2}}{2} \lambda_{\text {max }}(R) .
\end{aligned}
$$

Proof. Choose the following Lyapunov function: $V(t)=$ $V_{1}(t)+V_{2}(t)$; we can easily get the conclusion by the similar method which is used in Theorem 5 . results.

The following lemma can help us obtain the further

Lemma 8 (see [16]). Let $\Upsilon(t)>0(t \in \mathbb{R}), \tau(t) \in[0, \infty)$, $t_{0} \in \mathbb{R}$. Suppose that $D^{+} \Upsilon(t) \leq-\alpha \Upsilon(t)+c\left(\sup _{t-\tau(t) \leq s \leq t} \Upsilon(s)\right)$, $\forall t>t_{0}$. If $a>c>0$, then $\Upsilon(t) \leq \sup _{-\tau(t) \leq s \leq 0} \Upsilon\left(t_{0}+s\right) e^{-\varsigma\left(t-t_{0}\right)}$, $\forall t>t_{0}$, in which $0<\varsigma<a$ is determined by the equation $\varsigma-a+c e^{\varsigma \tau(t)}=0$. 
Corollary 9. Suppose that (A2) holds; the communication topology $G_{\sigma}$ satisfies (A3) and (A4). If there exist positive definite matrices $P, Q$ and a constant $\beta>0$ such that

$$
\begin{aligned}
& P\left(A+B^{\sigma}\right)+\left(A+B^{\sigma}\right)^{\mathrm{T}} P \leq-\beta I_{n}, \quad \sigma \in \wp, \\
& 2 h^{2} \frac{\lambda_{\text {max }}(P)}{\lambda_{\text {min }}(P)} \max _{\sigma \in \mathfrak{\wp}}\left\|\left(P B^{\sigma}\right)^{\mathrm{T}} Q^{-1} P B^{\sigma}\right\| \\
& \quad\left(\left\|A^{\mathrm{T}} A\right\|+\max _{\sigma \in \mathfrak{\wp}}\left\|\left(B^{\sigma}\right)^{\mathrm{T}} B^{\sigma}\right\|\right)+\lambda_{\text {max }}(Q)<\beta
\end{aligned}
$$

then, for the dynamical network (5), manifold $\Gamma_{1}$ and manifold $\Gamma_{2}$ are exponential stable.

Proof. Define a Lyapunov-Razumikhin function $V(t)=$ $V_{1}(t)$. According to Leibniz-Newton formula, we have

$$
\varepsilon(t)+\varepsilon(t-\tau(t))=\int_{t-\tau(t)}^{t} \dot{\varepsilon}(s) d s
$$

Then, (22) can be written as

$$
\dot{\varepsilon}(t)=\left(A+B^{\sigma}\right) \varepsilon(t)-B^{\sigma} \int_{t-\tau(t)}^{t} \dot{\varepsilon}(s) d s .
$$

According to (44), we have

$$
\begin{aligned}
D^{+} V(t)= & \varepsilon^{\mathrm{T}}(t)\left(P\left(A+B^{\sigma}\right)+\left(A+B^{\sigma}\right)^{\mathrm{T}} P\right) \varepsilon(t) \\
& -2 \int_{t-\tau(t)}^{t} \varepsilon^{\mathrm{T}}(t) P B^{\sigma} \dot{\varepsilon}(s) d s .
\end{aligned}
$$

It is noted that, for any positive definite matrix $\chi$, there is

$$
2 a^{\mathrm{T}} b \leq a^{\mathrm{T}} \chi a+b^{\mathrm{T}} \chi^{-1} b .
$$

Let

$$
\begin{aligned}
D^{+} V(t) \leq & -\beta \varepsilon^{\mathrm{T}}(t) \varepsilon(t)+\varepsilon^{\mathrm{T}}(t) Q \varepsilon(t) \\
+ & \left(P B^{\sigma} \int_{t-\tau(t)}^{t} \dot{\varepsilon}(s) d s\right)^{\mathrm{T}} \\
& \cdot Q^{-1}\left(P B^{\sigma} \int_{t-\tau(t)}^{t} \dot{\varepsilon}(s) d s\right) .
\end{aligned}
$$

From (41), we have

$$
\begin{aligned}
& D^{+} V(t) \leq-\beta \varepsilon^{\mathrm{T}}(t) \varepsilon(t)+\varepsilon^{\mathrm{T}}(t) Q \varepsilon(t) \\
& +\left(P B^{\sigma} \int_{t-\tau(t)}^{t} \dot{\varepsilon}(s) d s\right)^{\mathrm{T}} Q^{-1}\left(P B^{\sigma} \int_{t-\tau(t)}^{t} \dot{\varepsilon}(s) d s\right) \\
& \leq\left(-\beta+\lambda_{\max }(Q)\right) \varepsilon^{\mathrm{T}}(t) \varepsilon(t) \\
& +\max _{\sigma \in \mathfrak{Q}}\left\|\left(P B^{\sigma}\right)^{\mathrm{T}} Q^{-1} P B^{\sigma}\right\| \int_{t-\tau(t)}^{t} \int_{t-\tau(t)}^{t} \dot{\varepsilon}(s) \\
& \cdot \dot{\varepsilon}(r) d s d r \leq\left(-\beta+\lambda_{\max }(Q)\right) \varepsilon^{\mathrm{T}}(t) \varepsilon(t)+2 h^{2} \\
& \cdot \max _{\sigma \in \mathfrak{F}}\left\|\left(P B^{\sigma}\right)^{\mathrm{T}} Q^{-1} P B^{\sigma}\right\| \\
& \cdot\left(\left\|A^{\mathrm{T}} A\right\|+\max _{\sigma \in \mathfrak{V}}\left\|\left(B^{\sigma}\right)^{\mathrm{T}} B^{\sigma}\right\|\right) \times \sup _{t-2 \tau(t) \leq s \leq t} \varepsilon^{\mathrm{T}}(s) \\
& \cdot \varepsilon(s) \leq-\frac{\beta-\lambda_{\max }(Q)}{\lambda_{\max }(P)} V(t)+2 h^{2} \\
& \cdot \max _{\sigma \in \mathfrak{F}}\left\|\left(P B^{\sigma}\right)^{\mathrm{T}} Q^{-1} P B^{\sigma}\right\| \\
& \cdot\left(\left\|A^{\mathrm{T}} A\right\|+\max _{\sigma \in \mathfrak{Q}}\left\|\left(B^{\sigma}\right)^{\mathrm{T}} B^{\sigma}\right\|\right) \\
& \quad \times \frac{\sup _{t-2 \tau(t) \leq s \leq t} V(s)}{\lambda_{\min }(P)} .
\end{aligned}
$$

According to Lemma 8 , there exists $\eta>0$, such that $V(t) \leq \sup _{-2 \tau(t) \leq s \leq 0} V\left(\varepsilon\left(t_{0}+s\right)\right) e^{-\eta\left(t-t_{0}\right)}, t \geq t_{0}$. It is obvious that

\section{$\|\varepsilon(t)\|$}

$$
\leq\left(\frac{\lambda_{\max }(P)}{\lambda_{\min }(P)}\right)^{1 / 2} \sup _{-2 \tau(t) \leq s \leq 0}\left\|\mathcal{E}\left(t_{0}+s\right)\right\| e^{-(1 / 2) \eta\left(t-t_{0}\right)},
$$

$$
t \geq t_{0}
$$

That is, (22) is exponential stable for zero solution. Therefore, for the dynamical network (5), manifold $\Gamma_{1}$ and manifold $\Gamma_{2}$ are exponential stable.

If the Laplacian matrix $L^{\sigma}$ satisfies (A3) and (A4), then $L^{\sigma}$ has a zero eigenvalue; other eigenvalues have positive real parts. Let $L_{*}^{\sigma}=\left(L^{\sigma}+\left(L^{\sigma}\right)^{\mathrm{T}}\right) / 2$, where $L_{*}^{\sigma}$ is the Laplacian matrix of mirror strongly connected graph of $G_{\sigma}$ [5]. Therefore, $L_{*}^{\sigma}$ has one zero eigenvalue; other eigenvalues have positive real parts. Let $S^{-\mathrm{T}} L_{*}^{\sigma} S^{-1}=\operatorname{diag}\left[0, \bar{L}_{*}^{\sigma}\right]$; then $\bar{L}_{*}^{\sigma}$ is a positive definite matrix.

Corollary 10. Suppose that (A2) holds, the communication topology $G_{\sigma}$ satisfies (A3) and (A4). If b, $k>0$, such that

$$
\frac{k^{2}}{b^{2}} \lambda_{\max }\left(M_{e}\right)+\frac{1}{4 k \min _{\sigma \in \mathfrak{\wp}} \lambda_{\min }\left(\bar{L}_{*}^{\sigma}\right)}<1,
$$

where $h$ is small enough, then, for the dynamical network (5), manifold $\Gamma_{1}$ and manifold $\Gamma_{2}$ are exponential stable. 
Proof. Define a Lyapunov-Razumikhin function $V(t)=$ $V_{1}(t)$, where

$$
P=\left[\begin{array}{cc}
I_{(n-1) m} & \frac{k}{b} M_{e} \\
\frac{k}{b} M_{e} & M_{e}
\end{array}\right]
$$

According to Schur complement lemma and (50), we know that matrix $P$ is positive definite. We use the method that is similar to Corollary 9; then we have

$$
\begin{aligned}
& D^{+} V(t)=-\varepsilon^{\mathrm{T}}(t) Q \varepsilon(t)-2 \int_{t-\tau}^{t}\left(\varepsilon^{\mathrm{T}}(t) P B^{\sigma} A \varepsilon(s)\right. \\
& \left.\quad+\varepsilon^{\mathrm{T}}(t) P B^{\sigma} B^{\sigma} \varepsilon(s-\tau(t))\right) d s
\end{aligned}
$$

where

$$
Q=\left[\begin{array}{cc}
\frac{2 k^{2}}{b} \bar{L}_{*}^{\sigma} & 2 k \bar{L}_{*}^{\sigma}-I_{(n-1) m} \\
2 k \bar{L}_{*}^{\sigma}-I_{(n-1) m} & 2 b \bar{L}_{*}^{\sigma}-\frac{2 k}{b} M_{e}
\end{array}\right] .
$$

According to Schur complement lemma and (50), we know that matrix $Q$ is positive definite. After a simple calculation, we have

$$
\begin{aligned}
D^{+} V(t) & \\
\leq & \left(-\min _{\sigma \in \mathfrak{\wp}} \lambda_{\min }(Q)+h \max _{\sigma \in \mathfrak{\wp}}\left(\left\|P B^{\sigma}\right\|\left(\|A\|+\left\|B^{\sigma}\right\|\right)\right)\right) \\
\cdot & \varepsilon^{\mathrm{T}}(t) \varepsilon(t)+h \max _{\sigma \in \mathfrak{\wp}}\left(\left\|P B^{\sigma}\right\|\left(\|A\|+\left\|B^{\sigma}\right\|\right)\right) \\
\cdot & \sup _{t-2 \tau(t) \leq s \leq t} \varepsilon^{\mathrm{T}}(s) \varepsilon(s) \leq-V(t) \\
\cdot & \frac{\min _{\sigma \in \mathfrak{\wp}} \lambda_{\min }(Q)-h \max _{\sigma \in \mathfrak{\wp}}\left(\left\|P B^{\sigma}\right\|\left(\|A\|+\left\|B^{\sigma}\right\|\right)\right)}{\lambda_{\max }(P)} \\
+ & \frac{h \max _{\sigma \in \mathfrak{\wp}}\left(\left\|P B^{\sigma}\right\|\left(\|A\|+\left\|B^{\sigma}\right\|\right)\right)}{\lambda_{\min }(P)} \sup _{t-2 \tau(t) \leq s \leq t} V(s) ;
\end{aligned}
$$

we take

$h$

$$
<\frac{\min _{\sigma \in \mathfrak{\wp}} \lambda_{\min }(Q)}{\max _{\sigma \in \mathfrak{r}}\left(\left\|P B^{\sigma}\right\|\left(\|A\|+\left\|B^{\sigma}\right\|\right)\right)\left(1+\lambda_{\max }(P) / \lambda_{\min }(P)\right)} .
$$

According to Lemma 8, we can get the conclusion.

Remark 11. Learning from Corollaries 9 and 10, the upper bound of permitted time delay is given by (42) and (55). Based on Corollary 9, we can use a special $P$ matrix to get Corollary 10. Therefore, Corollary 10 is a special case of Corollary 9.

\section{Simulation Results}

In this section the proposed theorems have been used for synchronizing the second-order nodes in the dynamical
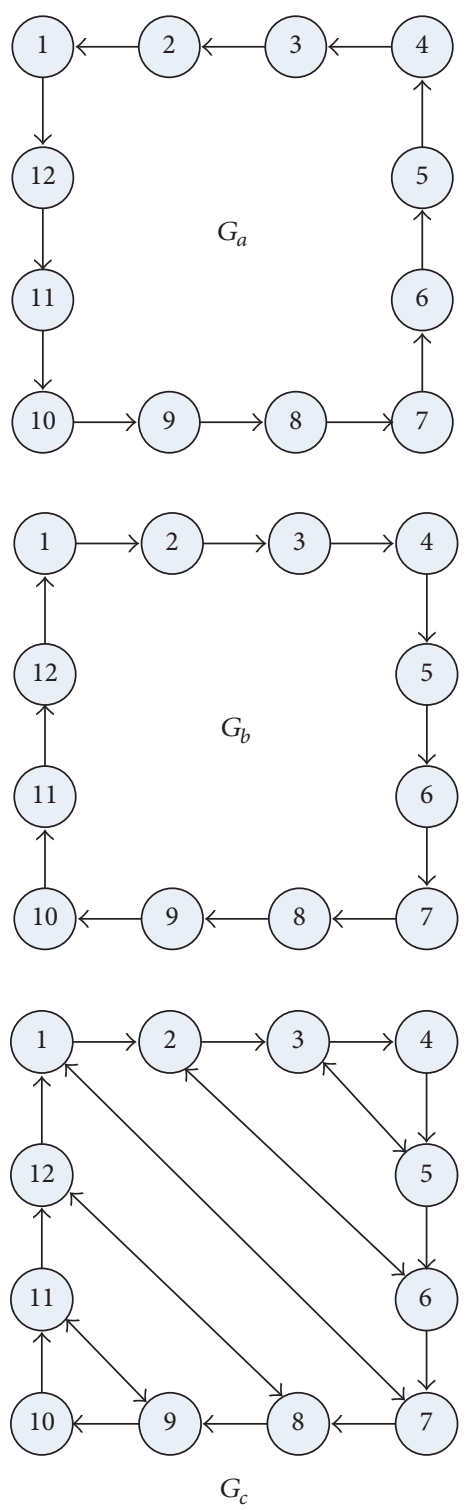

Figure 1: Strongly connected and balanced graphs.

network. Considering twelve second-order nodes in the dynamical network with switching topologies $\left\{G_{a}, G_{b}, G_{c}\right\}$, Figure 1 gives three strongly connected and balanced graphs with $0-2$ weights. The initial values of $x_{i}$ and $v_{i}$ are selected randomly in the regions $[0,800] \times[0,800]$ and $[0,800] \times$ $[0,800]$, respectively.

The inertia of twelve second-order nodes are $M_{1}=\operatorname{diag}\{1,1,1,1\}, M_{2}=\operatorname{diag}\{2,2,2,2\}, \ldots, M_{12}=$ $\operatorname{diag}\{12,12,12,12\}$. It is known from Theorem 5 that there exists a proper $h>0$ for any switching signal $\sigma(t)$ such that (22) is exponential stable for zero solution. Let $b=1$, $k=0.6, \alpha=0.3$, and $r=0.2$. Through the use of Theorem 5, the maximum upper bound of time delay $h=0.12$ can be acquired; the corresponding solutions are as follows. 
$P=\left[\begin{array}{cccccccccccc}0.0432 & 0 & 0.0095 & 0 & 0.0265 & 0 & 0.0179 & 0 & 0.0009 & 0 & 0.0245 & 0 \\ 0 & 0.0432 & 0 & 0.0095 & 0 & 0.0265 & 0 & 0.0179 & 0 & 0.0009 & 0 & 0.0245 \\ 0.0095 & 0 & 0.0239 & 0 & 0.0108 & 0 & 0.0091 & 0 & 0.0182 & 0 & 0.009 & 0 \\ 0 & 0.0095 & 0 & 0.0239 & 0 & 0.0108 & 0 & 0.0091 & 0 & 0.0182 & 0 & 0.009 \\ 0.0265 & 0 & 0.0108 & 0 & 0.0398 & 0 & 0.0068 & 0 & 0.007 & 0 & 0.0312 & 0 \\ 0 & 0.0265 & 0 & 0.0108 & 0 & 0.0398 & 0 & 0.0068 & 0 & 0.007 & 0 & 0.0312 \\ 0.0179 & 0 & 0.0091 & 0 & 0.0068 & 0 & 0.0188 & 0 & 0.0115 & 0 & 0.0123 & 0 \\ 0 & 0.0179 & 0 & 0.0091 & 0 & 0.0068 & 0 & 0.0188 & 0 & 0.0115 & 0 & 0.0123 \\ 0.0009 & 0 & 0.0182 & 0 & 0.007 & 0 & 0.0115 & 0 & 0.0462 & 0 & 0.0217 & 0 \\ 0 & 0.0009 & 0 & 0.0182 & 0 & 0.007 & 0 & 0.0115 & 0 & 0.0462 & 0 & 0.0217 \\ 0.0245 & 0 & 0.009 & 0 & 0.0312 & 0 & 0.0123 & 0 & 0.0217 & 0 & 0.0703 & 0 \\ 0 & 0.0245 & 0 & 0.009 & 0 & 0.0312 & 0 & 0.0123 & 0 & 0.0217 & 0 & 0.0703\end{array}\right]$,

$R=\left[\begin{array}{ccccccccccccc}0.1033 & 0 & 0.0044 & 0 & 0.0741 & 0 & 0.026 & 0 & -0.0063 & 0 & 0.053 & 0 \\ 0 & 0.1033 & 0 & 0.0044 & 0 & 0.0741 & 0 & 0.026 & 0 & -0.0063 & 0 & 0.053 \\ 0.0044 & 0 & 0.0213 & 0 & 0.003 & 0 & 0.0075 & 0 & 0.0209 & 0 & 0.0091 & 0 \\ 0 & 0.0044 & 0 & 0.0213 & 0 & 0.003 & 0 & 0.0075 & 0 & 0.0209 & 0 & 0.0091 \\ 0.0741 & 0 & 0.003 & 0 & 0.073 & 0 & 0.0146 & 0 & 0.0005 & 0 & 0.0593 & 0 \\ 0 & 0.0741 & 0 & 0.003 & 0 & 0.073 & 0 & 0.0146 & 0 & 0.0005 & 0 & 0.0593 \\ 0.026 & 0 & 0.0075 & 0 & 0.0146 & 0 & 0.023 & 0 & 0.0076 & 0 & 0.0191 & 0 \\ 0 & 0.026 & 0 & 0.0075 & 0 & 0.0146 & 0 & 0.023 & 0 & 0.0076 & 0 & 0.0191 \\ -0.0063 & 0 & 0.0209 & 0 & 0.0005 & 0 & 0.0076 & 0 & 0.0469 & 0 & 0.007 & 0 \\ 0 & -0.0063 & 0 & 0.0209 & 0 & 0.0005 & 0 & 0.0076 & 0 & 0.0469 & 0 & 0.007 \\ 0.053 & 0 & 0.0091 & 0 & 0.0593 & 0 & 0.0191 & 0 & 0.007 & 0 & 0.0808 & 0 \\ 0 & 0.053 & 0 & 0.0091 & 0 & 0.0593 & 0 & 0.0191 & 0 & 0.007 & 0 & 0.0808\end{array}\right]$,

$$
U=\left[\begin{array}{ccccccccccccc}
0.016 & 0 & 0.0013 & 0 & 0.011 & 0 & 0.006 & 0 & -0.0013 & 0 & 0.0091 & 0 \\
0 & 0.016 & 0 & 0.0013 & 0 & 0.011 & 0 & 0.006 & 0 & -0.0013 & 0 & 0.0091 \\
0.0013 & 0 & 0.001 & 0 & 0.0002 & 0 & 0.0028 & 0 & 0.0001 & 0 & 0.0022 & 0 \\
0 & 0.0013 & 0 & 0.001 & 0 & 0.0002 & 0 & 0.0028 & 0 & 0.0001 & 0 & 0.0022 \\
0.011 & 0 & 0.0002 & 0 & 0.0084 & 0 & 0.0009 & 0 & -0.0011 & 0 & 0.0042 & 0 \\
0 & 0.011 & 0 & 0.0002 & 0 & 0.0084 & 0 & 0.0009 & 0 & -0.0011 & 0 & 0.0042 \\
0.006 & 0 & 0.0028 & 0 & 0.0009 & 0 & 0.0174 & 0 & 0.0004 & 0 & 0.0134 & 0 \\
0 & 0.006 & 0 & 0.0028 & 0 & 0.0009 & 0 & 0.0174 & 0 & 0.0004 & 0 & 0.0134 \\
-0.0013 & 0 & 0.0001 & 0 & -0.0011 & 0 & 0.0004 & 0 & 0.0005 & 0 & -0.003 & 0 \\
0 & -0.0013 & 0 & 0.0001 & 0 & -0.0011 & 0 & 0.0004 & 0 & 0.0005 & 0 & -0.003 \\
0.0091 & 0 & 0.0022 & 0 & 0.0042 & 0 & 0.0134 & 0 & -0.0003 & 0 & 0.0121 & 0 \\
0 & 0.0091 & 0 & 0.0022 & 0 & 0.0042 & 0 & 0.0134 & 0 & -0.0003 & 0 & 0.0121
\end{array}\right] .
$$

We set $\tau(t)=0.2 \sin (3 t)+0.05$; Figures 2 and 3 show the numerical simulation results of twelve second-order nodes in the dynamical network with arbitrary switching signal. Figure 2 gives the inertial nodes' position error curves. Figure 3 


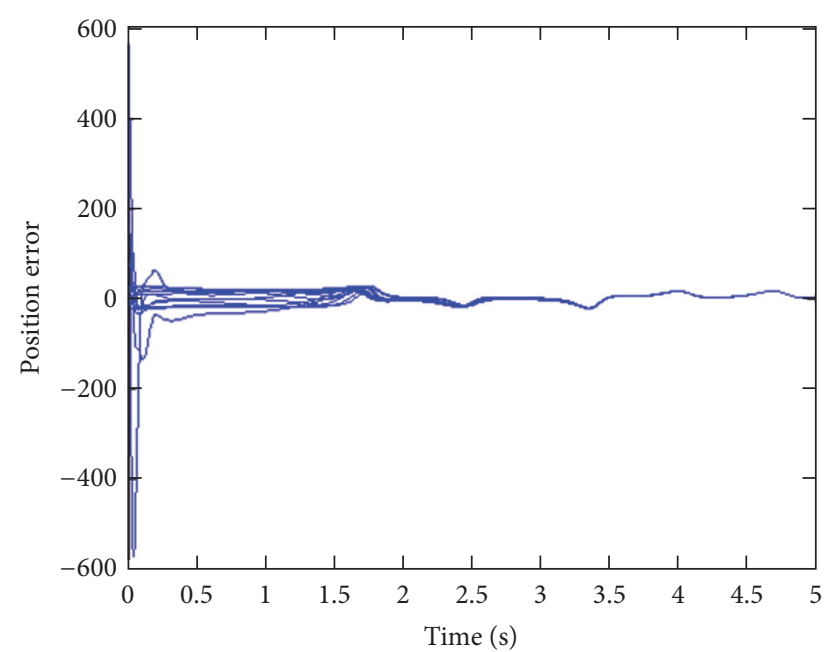

Figure 2: The inertial nodes' position error curves.

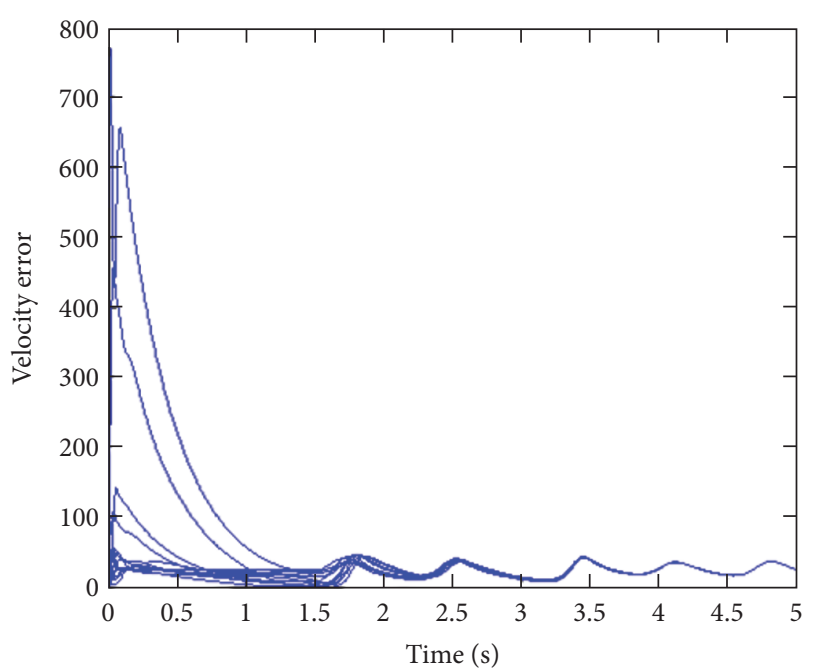

FIGURE 3: The inertial nodes' velocity error curves.

shows the inertial nodes' velocity error curves. It is obvious that for the dynamical network with communication time delay and switching topology, the control strategy achieves the exponential stability.

\section{Conclusions}

In this paper the exponential synchronization problem for second-order nodes in complex dynamical network with time-varying communication delays and switching communication topologies is investigated. Using the decomposition approach, a distributed control law has been designed such that the second-order nodes are exponential synchronized. The proposed method has been applied for synchronization of twelve second-order nodes, as a case study. Simulation results show the effective performance of the proposed control scheme. The results are expected to be more constructive in some practical control problems.

\section{Competing Interests}

This article content has no conflict of interests.

\section{Acknowledgments}

The authors would like to thank the reviewers for their very helpful comments and suggestions which have improved the presentation of the paper. The work of Miao Yu was supported by the National Natural Science Foundation of China (51407201), Doctoral Foundation of Beijing University of Civil Engineering and Architecture under Grant 00331614032, and Major State Basic Research Development Program of China (2012CB215206), respectively.

\section{References}

[1] C. Grabow, S. Grosskinsky, J. Kurths, and M. Timme, "Collective relaxation dynamics of small-world networks," Physical Review E, vol. 91, no. 5, Article ID 052815, 2015.

[2] M. Jalili, "Enhancing synchronizability of diffusively coupled dynamical networks: a survey," IEEE Transactions on Neural Networks and Learning Systems, vol. 24, no. 7, pp. 1009-1022, 2013.

[3] O. V. Maslennikov and V. I. Nekorkin, "Modular networks with delayed coupling: synchronization and frequency control," Physical Review E, vol. 90, no. 1, Article ID 012901, 2014.

[4] K. A. Newhall, M. S. Shkarayev, P. R. Kramer, G. Kovačič, and D. Cai, "Synchrony in stochastically driven neuronal networks with complex topologies," Physical Review E, vol. 91, no. 5, Article ID 052806, 2015.

[5] D. Xu, H. Zhang, and D. P. Mandic, "Convergence analysis of an augmented algorithm for fully complex-valued neural networks," Neural Networks, vol. 69, pp. 44-50, 2015.

[6] B. L. Parkin, P. J. Hellyer, R. Leech, and A. Hampshire, "Dynamic network mechanisms of relational integration," The Journal of Neuroscience, vol. 35, no. 20, pp. 7660-7673, 2015.

[7] V. H. Pereira, M. C. T. Gama, F. A. B. Sousa, T. G. Lewis, C. A. Gobatto, and F. B. Manchado-Gobatto, "Complex network models reveal correlations among network metrics, exercise intensity and role of body changes in the fatigue process," Scientific Reports, vol. 5, Article ID 10489, 2015.

[8] M. H. Matheny, M. Grau, L. G. Villanueva, R. B. Karabalin, M. C. Cross, and M. L. Roukes, "Phase synchronization of two an harmonic nanomechanical oscillators," Physical Review Letters, vol. 112, no. 1, Article ID 014101, pp. 461-467, 2014.

[9] A. Tavakoli, A. Cabello, M. Zukowski, and M. Bourennane, "Quantum clock synchronization with a single qudit," Scientific Reports, vol. 5, article no. 7982, 2015.

[10] S. Barbarossa and F. Celano, "Self-organizing sensor networks designed as a population of mutually coupled oscillators," in Proceedings of the IEEE 6th Workshop on Signal Processing Advances in Wireless Communications (SPAWC '05), pp. 475479, New York, NY, USA, June 2005.

[11] A. Jadbabaie, J. Lin, and A. S. Morse, "Coordination of groups of mobile autonomous agents using nearest neighbor rules," IEEE Transactions on Automatic Control, vol. 48, no. 6, pp. 988-1001, 2003.

[12] R. Shorten and F. R. Wirth, "On nonlinear AIMD congestion control for high-speed networks," in Proceedings of the IEEE Conference on Decision \& Control, pp. 633-638, December 2007. 
[13] Z. Lin, B. Francis, and M. Maggiore, "Necessary and sufficient graphical conditions for formation control of unicycles," IEEE Transactions on Automatic Control, vol. 50, no. 1, pp. 121-127, 2005.

[14] L. Moreau, "Stability of multiagent systems with time-dependent communication links," IEEE Transactions on Automatic Control, vol. 50, no. 2, pp. 169-182, 2005.

[15] W. Ren, R. W. Beard, and E. M. Atkins, "Information consensus in multivehicle cooperative control," IEEE Control Systems Magazine, vol. 27, no. 2, pp. 71-82, 2007.

[16] R. Olfati-Saber, J. A. Fax, and R. M. Murray, "Consensus and cooperation in networked multi-agent systems," Proceedings of the IEEE, vol. 95, no. 1, pp. 215-233, 2007.

[17] J. Hu and Y. Hong, "Leader-following coordination of multiagent systems with coupling time delays," Physica A, vol. 374, no. 2, pp. 853-863, 2007.

[18] W. Ren, "Consensus strategies for cooperative control of vehicle formations," IET Control Theory \& Applications, vol. 1, no. 2, pp. 505-512, 2007.

[19] W. Ren and E. Atkins, "Distributed multi-vehicle coordinated control via local information exchange," International Journal of Robust and Nonlinear Control, vol. 17, no. 10-11, pp. 1002-1033, 2007.

[20] Z. Tang, J. H. Park, and T. H. Lee, “Topology and parameters recognition of uncertain complex networks via nonidentical adaptive synchronization," Nonlinear Dynamics, vol. 85, no. 4, pp. 2171-2181, 2016.

[21] T. H. Lee, Q. Ma, S. Xu, and J. H. Park, "Pinning control for cluster synchronisation of complex dynamical networks with semiMarkovian jump topology," International Journal of Control, vol. 88, no. 6, pp. 1223-1235, 2015.

[22] K. Ramakrishnan, V. Venkatachalam, and G. Ray, "Comments on 'Improved delay-dependent stability criteria for continuous systems with two additive time-varying delay components," Communications in Nonlinear Science and Numerical Simulation, vol. 24, no. 1-3, pp. 1-3, 2015.

[23] J. Zhao, D. J. Hill, and T. Liu, "Synchronization of complex dynamical networks with switching topology: a switched system point of view," Automatica, vol. 45, no. 11, pp. 2502-2511, 2009.

[24] Y. G. Sun, L. Wang, and G. Xie, "Average consensus in networks of dynamic agents with switching topologies and multiple timevarying delays," Systems \& Control Letters, vol. 57, no. 2, pp. 175183, 2008.

[25] P. Lin and Y. Jia, "Average consensus in networks of multi-agents with both switching topology and coupling time-delay," Physica A: Statistical Mechanics and Its Applications, vol. 387, no. 1, pp. 303-313, 2008. 


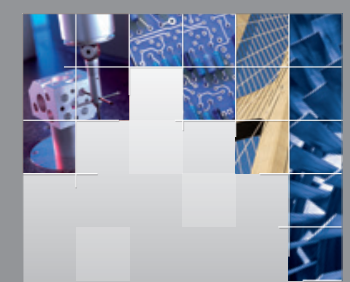

\section{Enfincering}
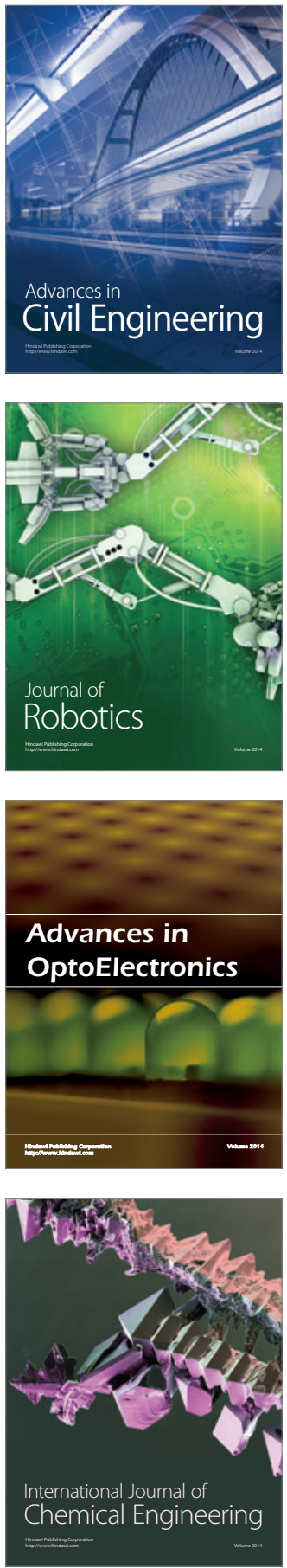

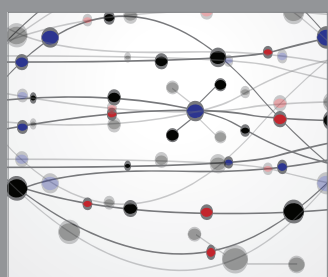

The Scientific World Journal

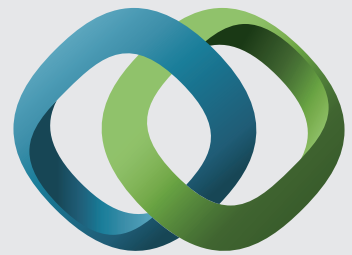

\section{Hindawi}

Submit your manuscripts at

https://www.hindawi.com
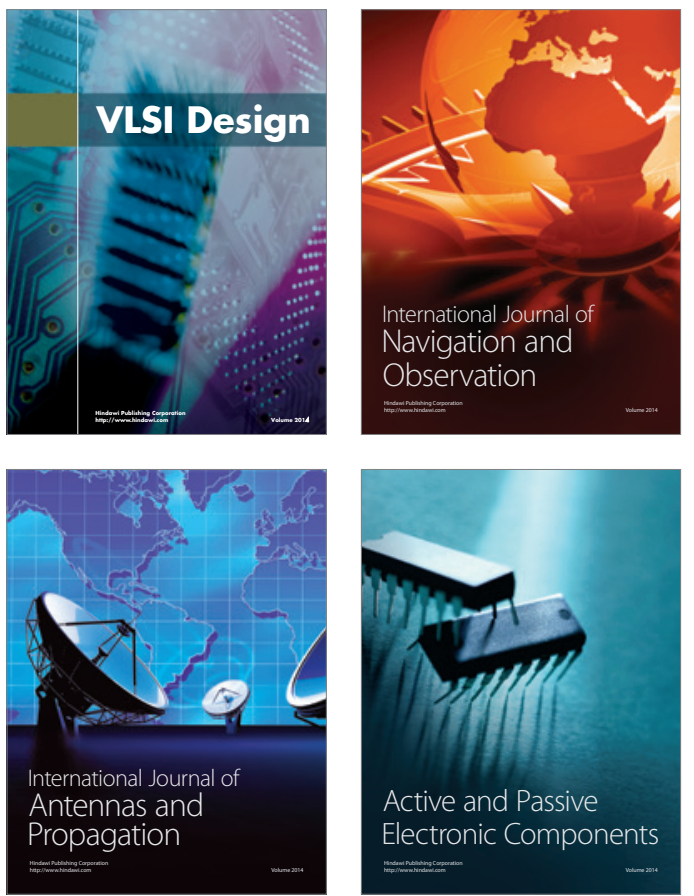
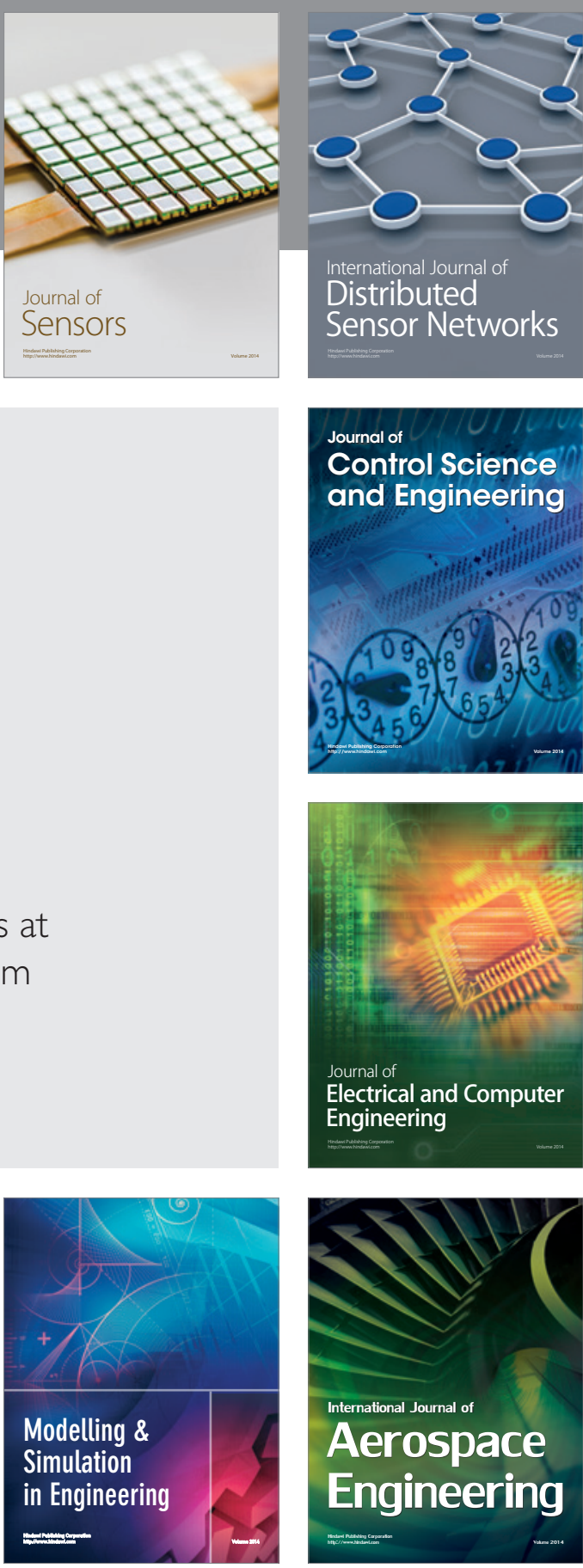

International Journal of

Distributed

Sensor Networks

$-$

Joumal of

Control Science

and Engineering
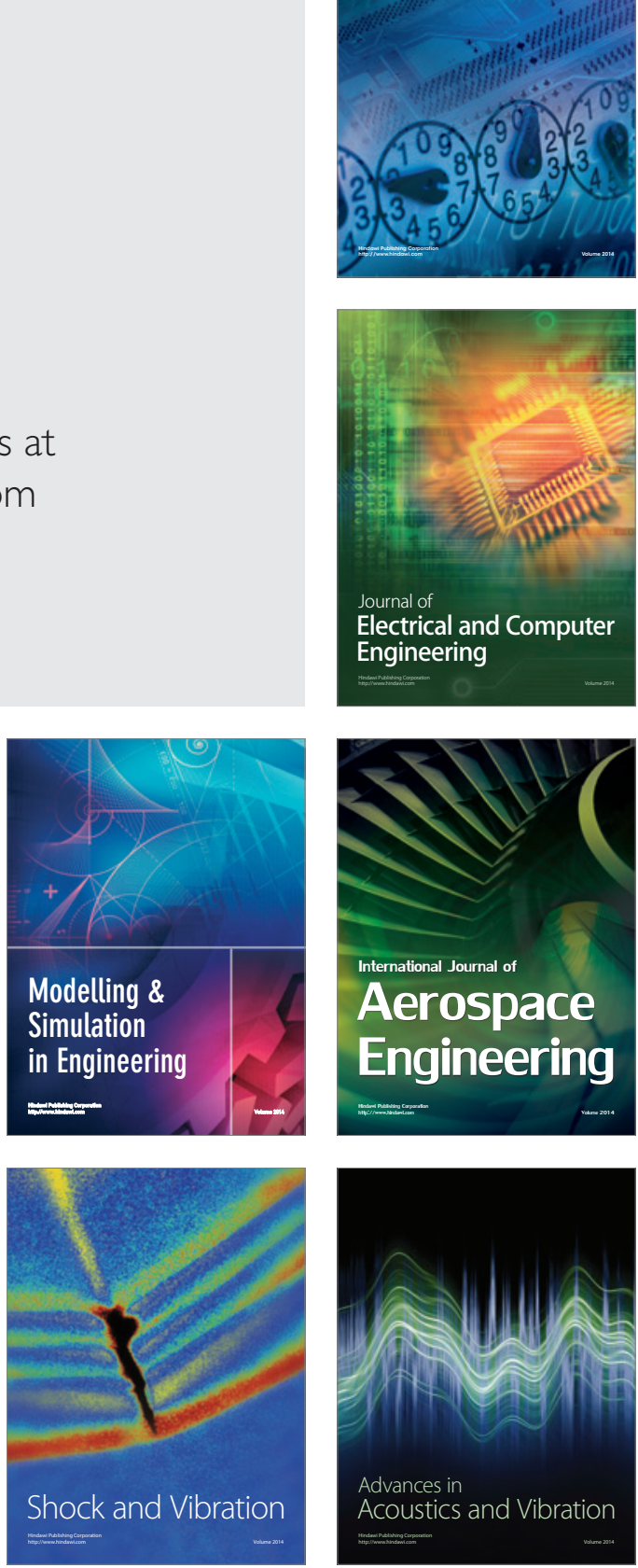